\title{
Thomas Hausmanninger
}

\section{Controlling the Net: Pragmatic Actions or Ethics Needed?}

\begin{abstract}
:
Do we need global ethics for the net? Is it even possible to put these into the form of a universal agreement, embodying the necessary rules and principles in an all-encompassing code of conduct? Or will any such endeavors simply shatter on the differences of cultures? Ought they be labeled as sort of attempted imperialism, more subtle perhaps in comparison with other forms of cultural imperialism-but nevertheless an attempt of such? If so, then ethical concepts need to be restricted to territorially or ethnically specific realms. In that case, the quest for Net-Ethics could perhaps be substituted by pragmatic actions: instruments of control that are simply technical and formal, devoid of moral input and moral convictions. Such a viewpoint has been offered lately in the form of a concept by the Bertelsmann Foundation, which combines rating and filtering instruments with a social lattice-work of net-supervision and transnational combinations of institutions of control. That concept indeed has its charm. It has its problems too; problems that can be made obvious from an ethical viewpoint and which counter the notion, that it is possible to supplant moral instances with pragmatic action. The text therefore reconstructs the concept in question, criticizes it and attempts to sketch an ethical approach to the problem that respects diversity and plurality.
\end{abstract}

\section{Agenda}

Introduction

Rating the Net

Why Rating and Filtering Cannot Suspend Ethics

Towards Ethics for the Net

\section{Author:}

Prof. Dr. Thomas Hausmanninger:

- University of Augsburg, Universitätsstraße 10, 86135 Augsburg, Germany

- $\widetilde{\widetilde{m}}+498215985820, ه$ thomas.hausmanninger@kthf.uni-augsburg.de, 昌 http://www.kthf.uniaugsburg.de/lehrstuehle/sozethik.shtml

- Relevant publications:

- Th. Hausmanninger (Ed.): Handeln im Netz. Bereichsethiken und Jugendschutz im Internet, München-Paderborn: W. Fink 2003 (= Schriftenreihe des ICIE 2). 


\section{Introduction}

Freedom cannot exist on its own. At the least, it must be enabled by restrictions against any attempt to use freedom to destroy its further existence (as happened for example in the German Weimarer Republik by the actions of the Nazis). Thus freedom-or to be more specific: the possibility of the universal sustainable use of freedom-has to be secured by measures of control. That seems to be necessary also in the case of freedom of communication and action on the Internet. Thus, in the last few years certain proposals have been presented to bring the Net under normative control. Most recently, concepts suggesting the control of Internet content in the form of a rating system and possibilities based on such ratings that would enable the filtering or blocking of specific content have received special attention. Concurrent with the discourse over the necessity and form of an Internet ethics-for example in the $\mathrm{ICIE}$-practical models und instruments for the normative regulation of Internet communication are therefore already available. The fact of availability seems to beg the question, whether an academic, ethical discourse appears to be even necessary at all, or whether such a discussion could be replaced simply by pragmatic controls-if this replacement has not already occurred.

Moreover, this question can be seriously supplemented with another: whether the path of rating, filtering and blocking would not eventually be the more favorable. Not least because of the Communitarianism debate, though in another context, has it become clear that ethical concepts-down even to foundational reflections-are culturally specific, or at the least that they cannot be conceived without certain culturally specific interests. This realization confronts the discussion about Internet ethics with its most trying difficulty: on the one hand, it should introduce a culturally-overlapping, generally acceptable proposal, while on the other hand it must unavoidably argue from culturally specific viewpoints, fundamental beliefs and preferences. From there, rating, filtering, and blocking could definitely offer a solution, if the corresponding possibilities were to be restricted to a particular cultural community, or, better yet, if the individual users would decide for themselves. Several different normative standards could be used in place of an Internet ethics; the use of which would make it superfluous, whether the standards followed certain ethical norms, or if they were based more on esthetic, functional, or educational theories. On-site pragmatic controls could not only substitute for the arduous, ethical discourse, but could also accomplish the following: they would be the functional equivalent of an Internet ethics, while simultaneously solving a problem that is most likely ethically impenetrable.

The quest for Internet ethics would then be obsolete, and the ICIE could then stop its endeavors in that field. But is the answer to the necessity of Netcontrol on behalf of its freedom that simple? May pragmatic action, devoid of universal moral principles and convictions, indeed supplant something like global ethics for the Net? In the following text I shall scrutinize that question by first recapitulating the concept(s) of rating and filtering, second pointing out its inherent and not sufficiently reflected problems and third proposing a concept for finding ways towards global ethics of the Net that at the same time do not destroy diversity and difference.

\section{Rating the Net}

Rating and filtering concepts have been offered at least since the 1990ies. For the establishment of an all-encompassing Net-control but two concepts (building on one another) seem specifically relevant. The first concept was outlined by Paul Resnick and James Miller (Resnick/Miller 1996), who through the World Wide Web Consortium (W3C) were participants in the development of the Platform for Internet Content Selection (PICS). Being a standard PICS does not profess to give any material criteria for filtering and blocking, but rather provides a formal structure, which can be filled with such criteria to a certain extent. Using PICS as a foundation, filtering and blocking programs, as well as labels for websites and documents, can be constructed: the labels are then read by the programs and according to the default selections, results are furnished. Thus, for instance, the Recreational Software Advisory Council (RSAC) has submitted a system of classification with material content-it works with the descriptors violence, nudity, sex, and language, which are further differentiated. Microsoft has built this classification system into its Internet Explorer on the foundation of PICS, allowing users access to the corresponding filters. That way, the selection of results comes one-sidedly from the filters. On one hand, the filters select according to negative lists of URLs which are put together by the software producers and block out those that contain the aforementioned descriptors. At the same time, websites and documents-often the title lines-are compared against a list of forbidden words, and selections are made in this way. Corresponding to their world view 
and cultural, esthetic, and moral attitudes, different nations, organizations, and groups should develop material criteria for labels as well as filtering and blocking programs. Labels should be provided from those that operate the websites or the authors of documents, yet third parties also should be able to offer suggestions. It should be possible, therefore, to characterize a website or document with several labels-even without the author's or operator's permission. As a result, at the label level different cultural and group-specific attitudes can be mirrored. This way, through blocking and filtering, Resnick and Miller intend to reproduce, and also simultaneously preserve, global plurality and the pluralism of modern, democratic societies (Resnick/Miller 1996).

The second concept builds off the first. Worked out by the Bertelsmann Stiftung in 1999, containing a high level of international cooperation, it was introduced in book form in 2000 (Memorandum 2000). The concept is distinctive in that it takes Resnick and Miller's proposal and develops it further into a mechanism combining technical, social, and institutional controls. First of all, the plan for the technical component is for the authors of content on the Internet-and not third parties-to provide labels according to a basic keyword list (Memorandum $2000,46)$. On the basis of this same list, different organizations and groups-churches for exampleshould be able to draw up templates: that is combinations of words, whose permissibility or impermissibility would be assigned in turn through various criteria-the protection of children and youth, moral conceptions, standards of esthetic taste, etc. Moreover, the templates should be supplemented by lists of individual websites, classified as either suitable or unsuitable, or even by additional filters, enabling a value-based, fine-tuning or intensification of the original filter (Memorandum 2000, 47). The selection process is then carried out in accordance with the value-templates and lists, whereby the users should be able to decide, which filters from which groups and organizations they would like to utilize. In order to enable a comprehensive filtering-process and to keep the demands on the competence of the users low, a filter of the user's choice should be installed with the purchase of the computer (Memorandum 2000,48 ).

At this technical level, the Internet is then embedded in the social monitoring through its users, which simultaneously should be provided institutional supports. This monitoring is focused in two ways: firstly, the users should test, whether they deem certain content to be illegal consulting the valid laws of their countries. Secondly in the case of legal content, they should weigh according to their discretion, whether an Internet site has been properly classified: that is, provided with the proper labels (Memorandum 2000, 51-61). The institutional supports then should provide this monitoring with power to carry out its findings. In order to enable self-control of the Internet, the concept advocates that each country establishes to this end: hotlines for service providers; the facilitation of selfmonitoring activities; rating and filtering organizations, etc. (Memorandum 2000, 52) In the course of this, hotline operators should cooperate with one another, both within their respective countries as well as internationally, and conclude a framework of various agreements to do this (Memorandum 2000, 33). In order to achieve the appropriate sanctioning power, for the area of legal content, it appears necessary that providers subjugate themselves to the procedures of self-regulation (Memorandum 2000 , 54). Concerning illegal content, the worldwide network of hotlines should work together with criminal prosecution agencies. Illegal content should be prosecuted always there, where the servers storing the data are located, according the laws of that country. Thus the concept envisions the establishment of much more an efficient, regulative control of the Internet, than any efforts to establish uniform, international regulations could ever provide.

\section{Why Rating and Filtering Cannot Suspend Ethics}

At first sight these concepts in fact appear to be able to form a functional equivalent to a concept of universal ethical standards for the Internet. The performance of multiple filters seems to take the place of binding, culturally overlapping decisions concerning values and norms. Consequently, Internet ethics would be superfluous, and a discussion about the (im)possibility of such would no longer be required. Yet, this first impression is deceptive. Not only do both concepts present a list of technical and organizational problems-which, unfortunately, cannot be handled in full here-but they also pose an ethical problem in themselves. Moreover, this problem is inherent to solutions of this nature. The problem is made apparent, when one (first) directs one's attention to the social processes out of which the concepts emerged.

These processes are not quite novel in the history of the media both in the US as well as in Germany (Hausmanninger 1992). As a rule, one runs into the 
situation, wherein a particular social group scandalizes a new medium of communication, because they hold either parts or the entire content thereof to be dangerous. The more effectively this group organizes itself and the more older, prevalent media absorb the expressed reservations, the greater the pressure will be at the political level to answer comprehensively the reservations with legal regulations. Usually, media enterprises attempt to get around such political rule-making by means of selfcensure in the form of self-control. The establishment of the American Comics Code of 1954 can be viewed as a virtual paradigm for this process; an analogy can be found in the development of the German Freiwillige Selbstkontrolle Fernsehen (television self control, FSF) in 1992. Normally supporters of self-control argue that it better serves the preservation of freedom of the media and communication than would governmental intervention, and, moreover, that the requirements of the democratic public-more correct: relevant groups-could be better realized thereby.

With regards to the Internet, this process was initiated in the United States through the debate concerning the presentation of sexual content, while the German discussion additionally focused itself on the offerings of right-wing extremists. The American discourse first led to a governmental reaction in the form of the Communications Decency Act of 1996; which, nevertheless, was seen as incompatible with the First Amendment by the Supreme Court and deemed unconstitutional in 1997. It is exactly in this political context that PICS were conceived as an alternative. German politics considered a similar institutional structure of control as has been established for monitoring the distribution of youthendangering material in the 1950ies, whereas that institutional structure should be completed by legal regulations for Internet providers (see Schily 2000). The Bertelsmann Concept is a response to this proposal. Resnick and Miller, as well as the Bertelsmann Stiftung, argue for rating, filtering and blocking as alternatives to governmental intervention. Analogous to older debates about the control of the media, they all focus their arguments on an individual's freedom of communication, which they claim must be protected. They see this as only being possible through the strengthening of the power of those utilizing communication-through a userempowerment-which is precisely what is made feasible by filtering technology (Resnick/Miller 1996; Memorandum 2000, 44).

Exactly by this argument, however, prove these concepts themselves to be at least partially deter- mined by normative preconceptions. With their focus an individual's freedom of communication, they place themselves, moreover, directly on an ethical basis of legitimacy. Over and above that, this basis is clearly culturally specific: the freedom of individuals to pursue their own concepts of happiness and with that their needs of communication, is an idea particular to western nationspredominantly found in Europe and North America. The idea belongs to the ethical focus of the project of modernity, specifically its emphasizing the subject's autonomy. It is, furthermore, directly connected with modernity's program of democratization which represents the political realization of freedom and autonomy. Therefore, both concepts are neither culturally independent nor do they form an ethically abstinent foundation for a plurality of standards. This option for plurality-specifically intercultural plurality-intricate to both concepts cannot be detached from thoughts of autonomy and the ethical core of modernity: only in and where autonomy is established, plurality can be substantially understood and delimited as a legitimate phenomenon. If a concept like that of the Bertelsmann Stiftung explicitly identifies the autonomy of individuals as such, the right to freedom of expression, and a variety of other ideas-that is a plurality-as the basis of the substantiation of said concept itself (Memorandum 2000, 44), it can be considered no longer a functional equivalent to ethics. On the contrary, that concept grounds its own necessity within the framework of a modern, ethical notion and attempts to develop its structure out of this ethical conception. Concepts like the one outlined above, then, do not make the question of an ethics of the Internet redundant, rather such a question shows itself as being implicit to the process itself. For here it is apparent that the question whether one or the other of the aforementioned concepts are capable of establishing a consensus is not merely a consensus pertaining to a pragmatic solution, but rather one that pertains at least just as much to confirming a consensus for the ethics contained therein.

Now one may wish to by and large subordinate the discussion of consensus-capability within German and American spheres-in spite of the clash over the boundaries of freedom in the debate between liberalism and communitarianism. Yet even in this case, the two concepts offer more problems than solutions-and here they are firmly ethical problems. The autonomy of individuals (standing as the principle upon which the concepts are based) and freedoms of the media and communication (which the concepts seek to protect) are actually endangered 
by these proposals. This comes first of all into effect starting with the fact that the autonomy of the users is not presented as the capacity of discernment regarding the Internet and its offerings; rather it is centrally relocated in the technical possibility of automated negative selection. Media competence hereby shrivels to the mere choice of a particular selection machine. This forms the most critical point of all, because-at least for the concept of the Bertelsmann Stiftung-not simply the safeguarding of children and youth is proposed, but also the shielding of adults from content which they do not wish to be confronted with (Memorandum 2000, 44). To achieve this, the self-protection from a link or web-address should not in any way require reflection, but should be accomplished automatically. Furthermore, this self-curtailing of maturity can go awry by the determinations of others, especially when the suggestion is followed to retain the default settings-chosen by a company-during the purchasing of the computer. Media competence, as reflective and discerning use of the media, is not furthered in this way, but rather its development is undermined and disabled.

Furthermore several more technically conditioned difficulties having disadvantageous effects can unfold. Labeling is essential for both proposals: that is the self-identification of websites and documents on the Internet by authors and operators. This, having to be realized with the provided basic vocabulary of 30 to 60 words (Balkin 2000, 266), means the introduction of a Procrustes' Bed into the process of description and the coercion of routine self-distortion; all the more so, since the vocabulary is to be split up into several criteria-like: content; genre; information desired by users; and the attributes utilized like text, pictures, animation, etc. (Waltermann 2000, 543)-and actually shrunk for characteristics like content. Similar distortions for the construction of filtering templates should be expected, if they work simply with a combination of these vocabulary elements. In this light, it appears questionable whether the rate of incorrect blockings-reaching up to $80 \%$ - of current filter systems can be minimized effectively (Haselton 2000, Weinberg 1997). Over and above that the question remains unanswered, how a basic vocabulary is possible in a transnational context: one which helps to avoid narrow, culturally specific notions as well as the domination of American conceptions of 'decency' (Chalaby 1998, 39). These possible distortions could disturb the fragile freedom of communication-especially when this occurs in the pre-installation phase due to a third party-against the wishes and beyond the notice of the individual users.

The dangers to freedom of communication are sharpened further by the foreseen hotline system proposed in the Bertelsmann concept. For example in the case of the German institution for monitoring the distribution of youth-endangering media (Bundespruefstelle fuer jugendgefaehrdende Schriften) only a certain few institutions and political representatives are invested with the protection of the youth-and, therefore, can file a legitimate grievance-whereas with the hotline all private citizens may lodge complaints. The German model concerning institutional monitoring of youthendangering material envisages a type of preselection, restricting possible grievances through expertise. Yet in the hotline system, all matters of folly and unknowledgeable interest can affect their influence on the process. The system, then, looks ethically precarious, because, in this case, those interested in the restriction of communication can achieve their end, while representing neither those affected (namely, those interested in using the Internet), nor even the majority of the population. The basis for this objection is to be found in the fact that in a system that exclusively focuses on the voices of those seeking limitations and demanding restrictions, those who do not desire such restrictions have no voice by definition. On behalf of freedom of the media it thus appears vital that calls for limitations of communication ought to be restricted and filtered, too.

Regarding the hotline system, this is not even the end of the story. Concurrently, the danger exists that marginal groups could use the system in order to push through their particular programs of restriction in an organized fashion. Such behavior is not unknown in the history of organized media criticism (Hausmanninger 1992; 2000). The strategic use of institutional and legal conditions, in this way, plays an increasingly serious roll. In the American sphere, the acronym SLAPP-Strategic Lawsuit Against Public Participation-has already been established to name such legally undesirable but hardly unavoidable conduct (UNESCO 1999, 75). The organized criticism of the media by marginal pressure groups poses problems even for the proposed self-control measures: where the emergence of complaints wins massive attention-relegating the masses interested in use of Internet offerings to silence-also grows the pressure to install self-control mechanisms, whose seriousness is proven through measures of limitation, which correspond to the complaints raised. Instead of the desired user-empowerment 
we may find the hegemonic influence of organized factions to impose restrictions. With regard to that possibility, the UNESCO fittingly speaks of a 'privatization of censorship' $(1999,74)$.

Lastly, it cannot be ruled out that the instruments for rating, filtering, and blocking could dovetail against the inherent and principal intention of both concepts and, by the use of governmental control, be used to completely abolish the autonomy of individuals. Feasibly, the PICS-platform and filtering system could be installed by Internet providers and imbued, for example, with political criteria. Unwanted and counter to their intention, both concepts actually provide instruments for non-democratic political systems. Seen through these objections, the proposals fall into what discourse ethics, in reliance upon linguistic pragmatics, calls a contradiction in performance-a contradiction between intended content and actual conduct: in this case, between the goal of the concept and the possible effects of its implementation.

\section{Towards Ethics for the Net}

Rating, filtering, and blocking alone cannot replace Internet ethics. Additionally, despite the objections outlined above, ethical regulation does not appear superfluous. Even where we are not forced to decide on a particular ethical theory for the systematic foundation needed for this regulation, the above sections make us aware of the factual necessity of such. Yet all this begs the question, in which way such a regulation can be reached. In order to answer this question, several requirements for such a regulation have to be kept in mind.

First, any ethical regulation of the Internet must correspond to the plurality and diversity of the world's cultures and notions of morality. Were this not the case, the regulation would tend towards cultural imperialism on a theoretical level, while practically it could not be implemented in the decentralized structure of the Internet. Especially the American and European cultures ought to be quite sensitive towards culturally imperialistic moves: They all embody not only the right to freedom of individuals and groups but more so the right for these individuals and groups to differ in a free and unmolested way from one another. Diversity and plurality are nothing less than the cornerstones of their free societies. Specifically European and American Philosophy furthermore has-under the influence of deconstructionism-developed a sense for difference and its significance in any concept of justice. Respecting difference thus has become a vital element of morality. Any concept negating the diversity and opting for forced uniformity would thus appear morally precarious. Contemporary American and European philosophy therefore should not seek solutions in the realm of information ethics opposed to difference and plurality. These solutions could not be sustained very long, either. Where an ethical concept does not respect and incorporate plurality and diversity, where it does not bespeak the various convictions of those individuals who are to live with it, looms the threat of its being subverted and undermined. Regarding the Internet, this result obtaining is especially plausible. On the other hand, an ethical concept must be capable of some consensus. That is why the outlined concepts of rating, filtering, and blocking also rely upon the readiness of individuals to restrict their behavior themselves and their corroboration in the institutional realization of censorship, while not relying upon a purely institutional carrying out thereof. As the history of the repressive protection of youth demonstrates, in this case, repression may alter the ways of obtaining certain banned media products, but not really their acquisition and reception by young people in a sufficient manner and to a sufficient degree (Vogelgesang 1991). (For the same reason, of course, the aforementioned danger of the censuring use of filtering instruments by non-democratic states is a bit moderated.) The necessary consensus therefore needs to be of a quality, that takes difference and diversity into account and makes them part of the consensus itself.

Second, the ethical regulation of the Internet must correspond to its various spheres of communication and activity, which form a diversity of its own. Norms for political discourse and the actions of NGOs differ from those needed for the mating and dating in Internet chatrooms. These, in turn, must be differentiated from the norms needed for economic communications and transactions, etc. The more the functions of the Internet differentiate themselves along the lines of social systems-and develop this differentiation further-the stronger the demand will become for the development Internetspecific ethics for each realm. This feature is, by the way, neither covered by rating and filtering, nor do the proposals even intend to address it. Third, the ethical regulation of the Internet requires transnational, legal support: above all, in enabling the effective control of illegal content and other actions. Fourth, and finally, Internet ethics must be supplemented by a Net-external non-repressive, educationally oriented youth protection, aimed at the 
generation of individual and autonomous media competence.

Attempts to establish ethical regulations that would take into account the plurality and diversity of the global nature of the Internet can, first of all, take cues from endeavors to ground similar notions of morality. Despite all the diversity and differences of cultures it appears possible to note some basic similarities in the multitude of ethical convictions around the globe. On empirical grounds, that has been shown for example by Hans Küng (1997) and his Project World Ethos: obviously the diverse ethical concepts converse in some basic norms, values and rules-even though these may not always have the same significance or authority in these various concepts. Similar to the endeavors in the field of substantiating basic human rights, Küng's efforts show, that it is apparently simpler to locate comparable moral norms than identical or comparable discourses for the substantiation of these norms. (Whereas from a European viewpoint it certainly appears possible to propose a metatheory to explain these convergences, and it may even be feasible to ground this in the self-reflection of a rational, moral self-consciousness [Hausmanninger 1994]. But this European viewpoint is not necessarily transposable into any other culture.). So, in order to generate a convergence of foundational ideas about norms, the unification of all theoretical conceptions is not really essential-the ways of justification and substantiation may remain plural whereas the convergences pose sort of a substantiation and justification of its own.

The reasons for this become clearer when considering the varied justifications and substantiations of human rights; to this end, advocates can utilize a wide range of religious and ethical lines of argumentation including: Kantian, subject-oriented; discourse ethical; justice-based; utilitarian; contract theoretical; or neo-Aristotelian approaches. Efforts to organize similar basic moral conceptions can be further developed into something like John Rawls' overlapping consensus. This forms the starting-point for a world ethos (Weltethos) of the Internet. The term 'overlapping' on the other hand takes into account that the ethos need not be of a 'monolithic' sort. On the contrary it ought to be sort of a partially connected, partially overlapping and partially discrete nature. Furthermore the overlapping part may be of different urgency and relevance in different cultures. Thus the world ethos of the Internet could form in itself sort of a net-and thus embody difference, diversity and plurality. Looming conflicts between the discrete parts of it could be softened by the regional character of these, which is made possible also on the net because of its segmentation (see below). At the same time this diverse and internally plural ethos can be considered sound, if it allows for the ordering of foundational ethical reflections. Each part of the ethos needs to be able to substantiate its form and content and thus be potentially criticizable. The intersection of the sundry ethical conceptions could be viewed as something like the core of the world ethos of the Internet. Analogous to the process through which the truth content of religious notions is strengthened by their convergence in several religions-the more instances the better-and anchored in their rational reflective concepts, that intersection attests to the especially well-grounded rightness of that core and also the related ethical convictions.

By looking for an overlapping consensus furthermore a normative instance is gained which allows for the introduction of the ethically fundamental difference between moral and immoral: concepts that directly oppose the core cannot be called ethical anymore. Thus the difference between legitimate and illegitimate elements of plurality and diversity can be noted. Immoral notions can be labeled as such and will not-as with them the ideologies of totalitarian systems-find a place in this overlapping consensus. They would compromise the possible establishment of a consistent, ethical foundation; not least because they are inclined to resort to methods of repression, terror, and torture in order to maintain their existence. Thus these systems demarcate the boundary of legitimacy for plurality and diversity. At the same time, the process leading to this world ethos of the Internet could ultimately play a roll in transnational processes, which could lead to the final disappearance of said political systems.

The factually given segmentation of communication on the Internet could itself be made fruitful for the search for this ethos and also for the creation of its specific, diverse and plural concretions. The entire world is not in point of fact in communication with itself on the Internet, but rather the communication falls into several strands. In contrast to other media of communication to this point, Internet communication tends to have a much stronger international component. Moreover, while the potential for the global networking of communication always exists, there is also the chance that one strand will merge with another. Nevertheless, certain barriers stand in the way of this possibility; in part the same obstacles that cause the segmentation, as well as provide pathways for the channeling, of the streams of 
communication. Three such barriers appear especially relevant for our inquiry here: the multiplicity of languages and their written form; the IT- and telecommunications-infrastructure; and the dominant interests of users.

Admittedly, this segmentation of the Internet is not always helpful or desirable-in particular, when this is accompanied by the exclusion of poorer regions of the world from global communication. Yet, some elements of the segmentation are quite useful. The factor of segmentation, for example, brings AngloSaxon and democratic, human-rights oriented societies closer to each other. In this strand, an understanding concerning an ethos of human-rights can certainly be achieved without a lot of qualms over cultural imperialism. The effects of localization can support a focus on specifics-for example, on various African ethics-and on the one hand make this an issue for discussion, while on the other hand lend veracity to diverse and plural viewpoints. At the same time, however, this localization remains embedded in global communication and must not come across as ghettoization. Hereby, a tie-in with a world ethos of the Internet appears possible. The plurality of specific notions of morality, as well as the segmentation of them along the interests of the users, are both helpful to that end. By following the specific segmentations of the users along the lines of shared interests or/and ethnic, local etc. features it is possible to establish specific moral codes which belong to specific groups-for example religious groups-as 'group-morals' which may be the morals of even transnational communities, like (once again) religious communities. These may form specific communicative segments in which groupspecific moral convictions are heeded. At the same time, this process must, from the beginning, be compatible with a comprehensive ethical framework-namely, the world ethos of the Internet-and avoide ghettoization and other missteps. In the joining of this segmentation with the global-nature of the communication, the Internet even offers the chance to reach beyond previous efforts to establish an ethical framework for all of humanity-this global-nature is a constant challenge to be faced by every user, yet without denying them the privilege to greater specificity or even exit opportunities.

Not only the plurality, but also the differentiation of communication and action on the Internet must be taken into account. Ethical regulations that correspond to specific areas have to take their place along side these moralities. For instance, the increasing presence of business on the Internet makes necessary a business ethics for the Internet.
The already existent ethics for specific realms of action in the real world could be borrowed from in this case. Yet simultaneously, the Internet poses new difficulties: particularly regarding one's dealings with data and information-which are much easier to 'acquire' on the Internet than in the real world. As regards privacy policies, the groundwork has already been laid. Furthermore, the Internet offers prospects for the establishment of moral standards for global, economic activities-and for their reflected, ethical grounding-which until now have not been possible. This continuing failure to found a framework for global rules remains one of business ethics' central problems. This shortcoming can be partially substituted for at the ethical level or by institutional self-constraint-e.g. industry agreements, codes of conduct, etc. As Karl Homann and Franz BlomeDrees emphasize (1992, 131-135), such substitutions, however, are time and again confronted with the situation of competition: economic actions must be rent producing. The serious opportunities for moral forerunners are to be primarily found, where such substitutions are image-building and profitable (Homann/Blome-Drees 1992, 137).

Image is, however, a product of communication. For that reason, in communicative settings like the Internet, institutionalized self-constraint and ethosspecified actions have a greater chance of coming about. On the Internet, businesses and even entire industries can better explain and justify their policies and actions to consumers-even changing policies to fit consumer demands-building up their image and winning the preference of certain groups. Over and above that, the communicative space that is the Internet compels a closer relationship between companies and their moral policies, because the next firm is but a mouse click away. Not only does the comparability of offers grow, but the moral standards of economic activity do as well. The competition for the best morals that develops out of this can conveniently influence the global business ethics for economic exchange on the Internet, as well as have an impact beyond cyber-space. This goes similarly for other ethical spheres and the cultivation of moral standards: in science, religious communities, and suchlike.

The necessity of rules and laws in the real world, though, draws attention to the fact that the flourishing activity of all working together cannot be guaranteed by morals and ethics alone. Therefore, a comparable legal support for the moral and ethical regulation of activities and communication on the Internet appears necessary. The legal regulations of individual countries and cultures differentiate them- 
selves here, as does the perceived relationship between morality and law. Once again, we stand before a situation entailing plurality and difference. For this, the Bertelsmann concept provides an appropriate strategy, even if all of its elements are not found satisfactory. Global framework agreements are indispensable: especially those that incorporate the world's legal cultures with each other, while providing for the effective control of criminality. With this, the basic idea at the beginning of the article comes into force: namely, the notion that the prosecution of punishable actions on the Internet or other means of communication should take place in, and within the legal structure of, the country wherein the actions took place or where the information is stored. From here, one can see the growth of avenues for bettered transnational cooperation. On the other hand, from an ethical perspective-even for illegal content-it is not always desirable that the most rigid notions of morality prevail. Let the difference between the democratic nations of Germany and the United States regarding the treatment of a traditional medium like film serve as an example: films that in Germany are banned, and have therefore been objects of criminal prosecution, in the USA have received a place in the Museum of Modern Arts. In such a case, the differences between the legal cultures can be viewed feasibly as a constructive provocation producing critical reflection about the more rigid regulation. For the reasons mentioned above, a hotline-system taking over the monitoring responsibilities for an area like illegal content appears problematic at best. It would be more appropriate to entrust this function, then as now, to the judicial and executive branches of government.

Lastly, we turn our attention to the problem of the protection of youth. One the one hand, the related anxieties overshoot the situation in reality to a certain degree here-up to now, a phenomenon which has accompanied all new forms of media. On the other hand, the endangerment of youth is not an ontologically-fixed, but rather a dynamic, culturally-specific concept. As above, the according actions to protect the youth should be oriented around the world's plurality and diversity, and their reproduction on the Internet. In addition, experience with regards to traditional media and endangerment of youth from extra-medial sources is waiting to be utilized. One of the central experiences, though one does not always eagerly take note of it, is the already mentioned fact that repressive means of protection-the overly protective educational attempt to guarantee a valuable socialization through the total absence of youth-endangering material- does not adequately lead to success. This insight has much longer been in force in media education theory, than in politics, law, and the institutions controlling social communication. Therefore, media education theory focuses foremost on teaching knowledgeable behavior. Regarding contact with certain forms of media this means supporting and encouraging the development of autonomous media competence. Institutions responsible for the areas of upbringing and education cannot achieve this alone. In upbringing, a culture must foster maturity: a maturity that needs to be anchored in the family, social groups, and religious and ethical communities. It is here, furthermore, that new models of the localization of civil society could prove useful, as their attention is directed more strongly toward the significance of the local quality of life for social togetherness. An upbringing that results in mature contact with media forms, then, a type of 'filter' of its own: albeit one that is more effective than the technical kind. It is a 'filter' that is embedded in the ethos of the individual and thus makes up an essential element of the capacity for self-selection of what is appropriate. Through this, many of the dangers that accompany certain offerings on the Internet can be more efficiently combated, than through repressive means. This fact may actually contribute to an easing of tensions: youth endangering subject matter neither makes up the bulk content on the Internet, nor did it appear for the first time in societies through the creation of the Internet. One has always had to impart lessons to children and youths-that they should not speak to strangers; that not every product of the media is meant and appropriate for consumption by young people; that lies and deceit come part and parcel with this world-so must they now also be taught how to deal with the Internet and its content. And as until now teaching children these other lessons has not been futile, we need not assume that the challenges of the present are insurmountable.

If these paths to moral standards for activities and communication on the Internet successfully develop and encourage media competence, then the more fascinating possibilities of the Internet can be further cultivated. Then as now, it is important to remember that the Internet is not something separate from the real world, but rather embedded in it. It then remains the responsibility of citizens of this real world to establish a structure enabling the flourishing of communication and other activities on the Internet.

Translation from German by Dominic Marcellino, Arkansas, partially rewritten by the author. 


\section{References}

Jack Balkin, Beth Noveck, Kermit Roosevelt (2000): Filtern von Internetinhalten - ein Best-PractisesModell, in: Waltermann 2000, 211-284

John Perry Barlow (1996): A Declaration of Independence of Cyberspace, in: http://hobbes.ucsa. uiuc.edu/sean/declaration.htm/

Rafael Capurro (2000): Strukturwandel der medialen Öffentlichkeit, in: http://www.capurro.de/zkmforum.htm

Jean Chalaby (1998): Protéger l'humanité contre la poursuite de faux dieux: un point de vue sociologique sur I'histoire de la censure, in: Michèle Paré, Peter Desbarats (Hg.): Liberté $d^{\prime}$ 'Expression et Nouvelles Technologies, ORBICOM/Canada (engl. Ausgabe : Freedom of Expression and New Communication Technologies)

Bennett Haselton (2000): Study of Average Error Rates for Censorware Programs, in: http://www. peacefire.org/error-rates/

Thomas Hausmanninger (1992): Kritik der medienethischen Vernunft, München

Thomas Hausmanninger (1994): "Diskurs Weltethos". Programmatische Notizen zur Diskussion um ein globales Rahmenethos, in: Catholica 4, 303-314

Thomas Hausmanninger (2000): Wider den guten Geschmack. Überlegungen zum Ausschluss der Betroffenen aus medienethischen Diskursen, in: medien praktisch Texte 3, 24-31

Karl Homann, Franz Blome-Drees (1992): Wirtschafts- und Unternehmensethik, Göttingen

Steven G. Jones (Ed. 1997): Virtual Culture: Identity and Communication in Cybersociety, London/Thousand Oaks/New Dheli

Hans Küng (1997): Weltethos für Weltpolitik und Weltwirtschaft, München/Zürich

Wilhelm von Ockham (1967/1970): Scriptum in librum primum sententiarum ordinatio, ed. $G$. Gál, S. Brown, in: Opera philosophica et theologica: Opera theologica I-II, St. Bonaventure NY

Paul Resnick, James Miller (1996): PICS: Internet Access Controls Without Censorship, in: http://www.w3.org/PICS/iacwcv2.htm

Howard Rheingold (1993): The Virtual Community: Homesteading on the Electronic Frontier, Reading, $M A$

Otto Schily (2000): Weltweite Kommunikation - eine Kultur gemeinsamer Verantwortung, in: Waltermann 2000, 83-102
Sherry Turkle (1999): Leben im Netz, Reinbek bei Hamburg

UNESCO (Hg. 1999): World Communication and Information Report, Paris

Verantwortung im Internet - Selbstregulierung und Jugendschutz. Memorandum der BertelsmannStiftung, in: Waltermann 2000, 27-62 (zit. als Memorandum 2000)

Waldemar Vogelgesang (1991): Jugendliche VideoCliquen, Opladen

Jens Waltermann, Marcel Machill (Hg. 2000): Verantwortung im Internet. Selbstregulierung und Jugendschutz, Gütersloh 